\title{
Hybridized Agile Software Development of Flight Control Team Tools for International Space Station's Payload Operations Integration Center
}

\author{
Cerese M. Albers, Ph.D., CAPM
}

QTEC Aerospace, Huntsville, AL, 35824, USA, cerese.m.albers@nasa.gov

Ground systems operations at the National Aeronautics and Space Administration's (NASA) Payload Operations and Integration Function (POIF) is increasing, via the High Operations Tempo (HOT) initiative, its ability to support more science activities with a fourth crew member on the International Space Station (ISS). The Flight Control Team's need to support this increased crew presence mandated the need for a series of software tools to better handle the increasing pace of payload science operations. The overall need was clear from the outset, but the full scope and user experience for each tool, were not as well understood, so establishing a fixed set of initial requirements was not feasible. An Agile Software Development (ASD) paradigm takes advantage of uncertainty, and plans for it, so it was deemed the most appropriate approach to create room for exploring novel concepts, and to mount a rapid and flexible response to inevitiably changing requirements. It facilitated the need for unprecedented collaboration between the Product Team (i.e. users from the Flight Control Team) and the Development Team (i.e. POIC systems engineers, developers, testers). This is a process shift in the development, test, and release of software from one that is prescriptive to one that is adaptive, which is necessary for these tools to have longevity. The application of ASD to the product development lifecycle permitted the timely incorporation of customer feedback, and, allowed for continuous quality improvements. This resulted in a suite of tools that are efficient, user-friendly, and enable POIF ground systems to support the increasing pace of payload science operations. ASD is not as much a set of prescriptive processes as it is a shift in mindset; one that moves from planning against change, to planning for change, thereby iteratively growing software towards user-defined value.

The Payload Operations and Integration Center (POIC) has successfully implemented ASD to complete work on the first three tools to support the fourth crew member operations. This was accomplished by employing a hybridized, Scrumderived, time-boxed Agile methodology. The main constraint for POIC is the rigid, biannual deployment cycle for ISS software syncing with onboard crew transitions, enforced because a software patch can be a major disruption to operations. Under normal circumstances, highly desired changes can take at least six months, but often over a year, before they are implemented in flight. Software defects occurring in operations are highly prioritized, however user requested changes are not as critical. Thus, with only 18 months to develop and deploy the initial three tools, a much faster way was needed to capture and address important user requests. It also opened the door to include human factors considerations of look and feel in the software design. From the outset, there were unique considerations to establishing an organized user feedback process. For one, the user community was predominantly composed of professionals on rotating shiftwork schedules, so coordinating user evaluations during normal business hours proved challenging. This was overcome by strategically planning Sprint lengths and providing event notifications, long in advance, to ensure adequate participation.

The process of obtaining feedback on the tools differs from other user evaluation approaches used by non-Agile projects developed by POIC, in that it is structured to minimize paperwork and turn-around time. Inputs from the Product Team are sought early and often, with the goal of creating reliable software that has both intrinsic (functional) and extrinsic (look, feel, experiential) quality. This process has been facilitated with the creation of a dedicated test environment for hosting the periodic release of Sprint-developed capabilities for assessment by the Product Team. During a user evaluation, the Product Team gets hands-on experience with the software in this test environment. A set of minimum success criteria drives high-level requirements for capabilities that are delivered each Sprint, but leaves the feature implementation details to the talent and technical expertise of the Development Team. These features are evaluated at strategic intervals in the user evaluation environment.

The application of the ASD paradigm to the POIC development lifecycle has brought about unprecedented levels of coordination and cooperation between POIC and POIF, resulting in the delivery of highly valuable products that best meet the needs of the ground systems community for payload science activities. The team-level cooperation has been facilitated by introducing regular mid-Sprint tag-ups, Agile retrospectives, socials, and team-building activities that other projects do 
not experience. The metrics taken before and after the development of the tools have shown large increases in productivity, situational awareness, error reduction, time savings, and the ability to communicate more efficiently. Additionally, the user community has indicated that they have appreciated the opportunity to interact with the software very early and have their feedback incorporated so readily on the tools' usage, look and feel. Likewise, developers report increased satisfaction in their work because it has been so positively received by the user community. These results have provided sufficient evidence of success such that the customer has requested the application of ASD for additonal POIFdesired fourth crew tools.

SpaceOps 2018 will bring together experienced professionals in ground systems engineering and data management, and opportunities will abound to exchange ideas and lessons learned for how to design, develop, and deploy tools to support science activities in space. This will be a one-of-a-kind opportunity to share success stories and glean insights from how other partners in the industry are developing tools to support an increasing pace of payload science operations. ASD is an optimal approach that offers higher return on investment and faster time-to-completion than traditional software development paradigms, while aiming at delivering high quality products and customer-driven value. 